\title{
Stochastic mixing model with power law decay of variance
}

\author{
By S. Fedotov, M. Ihme and H. Pitsch
}

\section{Motivation and objectives}

The mixing of a conserved scalar $c=c(t, \boldsymbol{x})$, advected by a turbulent flow, remains a problem of both fundamental and practical interest. One of the basic characteristics of the mixing process is the rate at which the scalar variance $\sigma_{c}^{2}(t)=\left\langle(c-\mu)^{2}\right\rangle$ decays with time. Here $\mu$ is the mean value, and the angular brackets $\langle\cdot\rangle$ denote an averaging procedure. One of the simplest and widely used mixing models is the interaction by exchange with the mean (IEM) (Villermaux \& Devillon 1972) or linear mean square estimate model (LMSE) (Dopazo \& O'Brien 1974, Sabel'nikov \& Gorokhovski 2001). In this model, the scalar relaxes toward its mean $\mu$ according to the equation

$$
\frac{\mathrm{d} c}{\mathrm{~d} t}=-\frac{1}{\tau}(c-\mu) \text {. }
$$

In the coalescence-dispersion model (CD) (Curl 1963), mixing of two particle volumes is described by (1) a coalescence and (2) dispersion process during one time step $\Delta t$. After that time, both particles have the same scalar value, which is equal to the mean of the two values before the mixing. The pdf relaxes to a bell-shaped distribution but deviates from the Gaussian shape primarily in the tails of the distribution (Peters 2000). Both models introduce a time scale $\tau$ which is commonly approximated by a turbulent time scale. Other mixing models are based on mapping closure, Fokker-Planck model, euclidean minimum spanning trees (EMST), or Langevin model. Reviews of these can be found in Peters (2000) and Pope (2000).

There are two different laws governing the decay rate of a passive scalar:

(i) the exponential law

$$
\sigma_{c}^{2}(t) \propto \exp \{-t / \tau\}
$$

with the characteristic time $\tau$ and

(ii) the power law

$$
\sigma_{c}^{2}(t) \propto t^{-\alpha}
$$

without any characteristic time scale (Lesieur 1997).

Most theoretical models introduce a characteristic time scale and assume implicitly or explicitly the exponential decay rate (1.2) which is only appropriate for stationary turbulence.

The main purpose of this paper is to study the mixing process following the power law (1.3) that is typical for decaying turbulence. Experimental results show that the decay of the variance strongly depends on the initial ratio of the velocity and scalar length-scales and that there is no universal decay exponent (Durbin 1982).

It is well known that the decay exponent $\alpha$ depends on the low wavenumber part of the scalar and velocity spectrum, $E_{c}(k, t)$ and $E(k, t)$ (Lesieur 1997). One can expand both spectra into a Taylor series: $E_{c}(k, t)=2 \pi k^{2}\left(C_{0}+C_{2} k^{2}+\mathcal{O}\left(k^{4}\right)\right)$ and $E(k, t)=$ 
$2 \pi k^{2}\left(B_{0}+B_{2} k^{2}+\mathcal{O}\left(k^{4}\right)\right)$ (Chasnov 1994). For high Reynolds and Peclet numbers, the scalar variance can be considered as a function of $C_{0}, B_{0}$ and $t$ alone and dimensional arguments lead to

$$
\sigma_{c}^{2}(t) \propto C_{0} B_{0}^{-3 / 5} t^{-6 / 5}
$$

with $\alpha=1.2$. For $C_{0}, B_{0}$ equal to zero, the scaling argument results in different approximate decay laws with $\alpha$ equals to $6 / 7,10 / 7$ or 2 (Lesieur 1997).

Here we present a simple stochastic mixing model based on the law of large numbers (LLN) (Feller 1966). The reason why the LLN is involved in our formulation of the mixing problem is that the random conserved scalar $c=c(t, \boldsymbol{x}(t))$ appears to behave as a sample mean. It converges to the mean value $\mu$, while the variance $\sigma_{c}^{2}(t)$ decays approximately as $t^{-1}$. Since the variance of the scalar decays faster than a sample mean (typically $\alpha$ is greater than unity), we will introduce some non-linear modifications into the corresponding pdf-equation (see Eq. (2.28) below). The main idea is to develop a robust model which is independent from restrictive assumptions about the shape of the pdf. Here we exploit the similarity of the behavior of a scalar $c$ to that of the sample mean

$$
c_{n}=\frac{1}{n} \sum_{k=1}^{n} \zeta_{k},
$$

where $\zeta_{1}, \ldots, \zeta_{n}$ is a sequence of mutually independent random variables, each having a mean $\mu$ and standard deviation $\sigma_{\xi}^{2}$. The LLN tells us that the random sum $c_{n}$ tends to the mean value $\mu$ with probability one, while the variance $\sigma_{c_{n}}^{2}=\left\langle\left(c_{n}-\mu\right)^{2}\right\rangle$ decays as $\sigma_{\xi}^{2} / n$. Then, any arbitrary initial pdf $p\left(n_{0}, c\right)$ tends to a $\delta$-distribution $\delta(c-\mu)$ as $n \rightarrow \infty$. In the present paper the discrete increment $n$ can be understood as the time variable $t$.

The main result of this paper is the derivation of the time-discrete non-linear integral equation for the pdf of $c$

$$
\begin{gathered}
p(t+1, c)=\int_{0}^{1} \int_{-1}^{\frac{1+t}{\lambda}-1} \frac{t+1}{1+t-\lambda(1+\varepsilon)} p\left(t, \frac{(t+1)(c-\mu)-\lambda(1+\varepsilon)(\widetilde{\eta}-\mu)}{1+t-\lambda(1+\varepsilon)}\right) \times \\
p(t, \widetilde{\eta}) \psi(t, \varepsilon) \mathrm{d} \varepsilon \mathrm{d} \widetilde{\eta},
\end{gathered}
$$

for $t=1,2,3, \ldots$. Here $\psi(t, \varepsilon)$ is the pdf for the exchange rate and $\lambda$ is the mixing intensity (see below). The main property of this equation is that it describes the relaxation from an arbitrary initial distribution to a $\delta$-function

$$
p(t, c) \rightarrow \delta(c-\mu) \quad \text { as } \quad t \rightarrow \infty
$$

and the decay of the variance, $\sigma_{c}^{2}(t)=\left\langle(c(t)-\mu)^{2}\right\rangle$, is of the form $t^{-\alpha}$. The case $\alpha=1$ corresponds to the law of large numbers.

The remainder of this paper is organized as follows. In Section 2 we derive the integral equation from a stochastic difference equation describing the evolution of the pdf of a passive scalar in time. The stochastic difference equation introduces an exchange rate $\gamma_{n}$ which we model in a first step as a deterministic function. In a second step, we generalize $\gamma_{n}$ as a stochastic variable taking fluctuations in the inhomogeneous environment into account. In Section 3 we solve the non-linear integral equation numerically and analyze the influence of the different parameters on the decay rate. The paper finishes with a conclusion. 


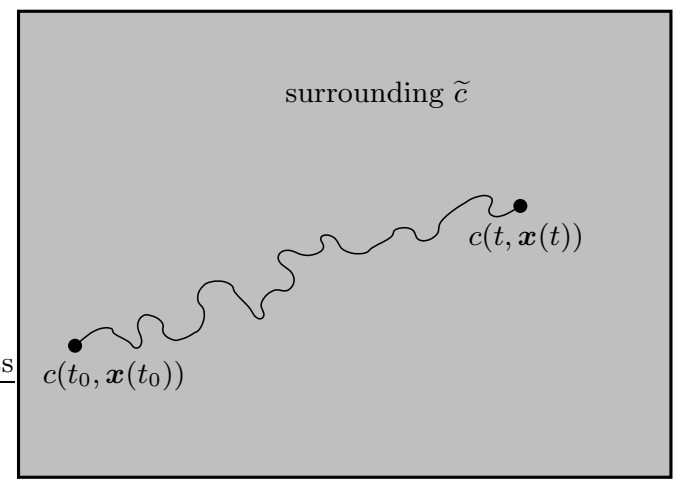

FiguRE 1. Mixing problem along Lagrangian path way.

\section{Mixing model}

\subsection{Problem statement}

The evolution of a passive scalar $c=c(t, \boldsymbol{x})$ is governed by the stochastic PDE

$$
\frac{\partial c}{\partial t}+\boldsymbol{v}(t, \boldsymbol{x}) \nabla c=D \nabla^{2} c,
$$

where $\boldsymbol{v}(t, \boldsymbol{x})$ is the random velocity field and $D$ is the molecular (or thermal) diffusivity. The classical problem is to derive a closed equation for the Euler one-point probability density function (pdf) $p=p(c ; t, \boldsymbol{x})=\langle\delta(c-c(t, \boldsymbol{x}))\rangle$. A detailed discussion of this, still unsolved, problem can be found in Pope (2000). In the present paper we consider the mixing problem in the Lagrangian framework by introducing a passive scalar $c(t)=$ $c(t, \boldsymbol{x}(t))$ of a particle volume moving with the velocity $\boldsymbol{v}(t, \boldsymbol{x}(t))$ (see Fig. 1).

\subsection{Stochastic difference equation}

The equation for the scalar $c(t)=c(t, \boldsymbol{x}(t))$ of the particle volume moving with the random velocity $\boldsymbol{v}(t, \boldsymbol{x}(t))$ can be approximated by the stochastic equation

$$
\frac{\mathrm{d} c}{\mathrm{~d} t}=-\gamma(t)(c-\widetilde{c}(t)),
$$

where the exchange rate $\gamma(t)$ and the ambient concentration $\widetilde{c}(t)$ are random processes. For the constant values of $\gamma$ and $\widetilde{c}$, we obtain the IEM model (1.1) with the exponential decay:

$$
\frac{c(t)-\widetilde{c}}{c(0)-\widetilde{c}}=\exp \{-\gamma t\} .
$$

The key feature of the present model is that $\widetilde{c}(t)$ is a random process and functional of $c(t)$ itself. The crucial assumption that relates Eq. (2.2) to the law of large numbers is that the mean value $\langle\gamma(t)\rangle$ behaves as $t^{-1}$ for large $t$. One can show that for a Gaussian scalar field $c=c(t, \boldsymbol{x})$, the mean value $\langle\gamma(t)\rangle$ is proportional to the ratio $\chi / \sigma_{c}^{2}$, where $\chi$ is the mean scalar dissipation rate, $\chi=2 D\left\langle(\nabla c)^{2}\right\rangle$ (Pope 2000). It follows from

$$
\frac{\mathrm{d} \sigma_{c}^{2}}{\mathrm{~d} t}=-\chi,
$$


that if $\sigma_{c}^{2}(t) \propto t^{-\alpha}$ then $\chi$ decays as $t^{-(1+\alpha)}$, and therefore $\langle\gamma(t)\rangle \propto t^{-1}$. The appearance of the power law decay can be understood if we assume that $\gamma=\lambda\left(t_{0}+t\right)^{-1}$

$$
\frac{\mathrm{d} c}{\mathrm{~d} t}=-\frac{\lambda}{t_{0}+t}(c-\widetilde{c}) \text {. }
$$

The solution to this simplified equation is

$$
\frac{c(t)-\widetilde{c}}{c(0)-\widetilde{c}}=\left(\frac{t_{0}}{t_{0}+t}\right)^{\lambda}
$$

Since our aim here is to relate the mixing problem to the law of large numbers, it is more convenient to rewrite Eq. (2.2) as a stochastic difference equation

$$
c_{n+1}-c_{n}=-\gamma_{n}\left(c_{n}-\widetilde{c}_{n+1}\right), \quad 0 \leq c_{n} \leq 1, \quad \text { for } n=1,2,3, \ldots
$$

where $\gamma_{n}$ and $\widetilde{c}_{n}$ are assumed to be sequences of mutually independent random variables with the densities

$$
\omega(n, \gamma)=\frac{\mathrm{d}}{\mathrm{d} \gamma} P\left\{\gamma_{n}<\gamma\right\}, \quad \phi(n, \widetilde{c})=\frac{\mathrm{d}}{\mathrm{d} \widetilde{c}} P\left\{\widetilde{c}_{n}<\widetilde{c}\right\}
$$

and the first moments

$$
\left\langle\gamma_{n}\right\rangle=\int_{0}^{1} \gamma \omega(n, \gamma) \mathrm{d} \gamma=\frac{\lambda}{1+n}, \quad\left\langle\widetilde{c}_{n}\right\rangle=\int_{0}^{1} \widetilde{c} \phi(n, \widetilde{c}) \mathrm{d} \widetilde{c}=\mu .
$$

Here the mean exchange parameter $\left\langle\gamma_{n}\right\rangle$ has been chosen in such a way that it is equal to $\lambda / 2$ at time $n=1$. The parameter $\lambda$ can be regarded as a measure of the mixing intensity. Since $0 \leq c_{n} \leq 1, \gamma_{n}$ obeys the inequality $0 \leq \gamma_{n} \leq 1$. By introducing the deviations from the mean $\mu$ for the concentration of the particle and its surrounding, respectively,

$$
\begin{aligned}
& u_{n}=c_{n}-\mu, \\
& \xi_{n}=\widetilde{c}_{n}-\mu
\end{aligned}
$$

we can rewrite Eq. (2.7) as

$$
u_{n+1}=u_{n}-\gamma_{n}\left(u_{n}-\xi_{n+1}\right), \quad-\mu \leq u_{n} \leq 1-\mu, \quad n=1,2,3, \ldots,
$$

where $\xi_{n}$ is a sequence of zero mean, independent random variables with the density $\phi(n, \xi)$ and $-\mu \leq \xi \leq 1-\mu$.

\subsection{Law of large numbers and forward Kolmogorov equation}

To illustrate the connection between the mixing problem and the law of large numbers, consider the case when the sequence $\xi_{n}$ is stationary, that is $\varphi(\xi)$ is independent of $n$, and the exchange parameter $\gamma_{n}$ is a deterministic sequence of the form

$$
\gamma_{n}=\frac{1}{1+n}
$$

with $\lambda=1$. If we assume $u_{1}=\xi_{1}$, it follows from Eq. (2.12) that $u_{2}=\left(\xi_{1}+\xi_{2}\right) / 2, u_{3}=$ $\left(\xi_{1}+\xi_{2}+\xi_{3}\right) / 3$ and so on. Therefore, the solution of the equation (2.12) can be written as a sample mean

$$
u_{n}=\frac{1}{n} \sum_{k=1}^{n} \xi_{k}
$$


which tends to the mean $\mu$ as $n \rightarrow \infty$ while the variance $\left\langle u_{n}^{2}\right\rangle \propto n^{-1}$.

The advantage of having an equation (2.12) is that we can easily derive an equation for the probability density function

$$
p(n, u)=\frac{\mathrm{d}}{\mathrm{d} u} P\left\{u_{n}<u\right\}, \quad n=1,2,3, \ldots .
$$

It follows from Eqs. (2.12) and (2.13) that $u_{n}$ is a discrete Markov processes and the pdf for this process satisfies the forward Kolmogorov equation (Feller 1966)

$$
p(n+1, u)=\frac{n+1}{n} \int_{-\mu}^{1-\mu} p\left(n, \frac{(n+1) u-\xi}{n}\right) \varphi(\xi) \mathrm{d} \xi, \quad n=1,2,3, \ldots
$$

Here we used the inverse equation

$$
u_{n}=n^{-1}\left[(1+n) u_{n+1}-\xi_{n+1}\right] .
$$

The main properties of the solution of the Kolmogorov equation (2.16) with the arbitrary initial condition $p(1, u)$ are

$$
p(n, u) \rightarrow \delta(u) \quad \text { as } \quad n \rightarrow \infty
$$

and

$$
\int_{-\mu}^{1-\mu} u^{2} p(n, u) \mathrm{d} u \rightarrow n^{-1} \quad \text { as } \quad n \rightarrow \infty .
$$

The asymptotic behavior of $p(n, u)$ for large $n$ is quite universal. It can be written as

$$
p(n, u) \propto \sqrt{n} \exp \{-n S(u)\} \quad \text { for } \quad n \gg 1,
$$

where the function $S(u)$ depends on the particular choice of the density $\varphi(\xi)$ for the random sequence $\xi_{k}$. One can show that $S(u)$ obeys the equation (Knessl et al. 1985)

$$
u \frac{\mathrm{d} S}{\mathrm{~d} u}-S=\ln M\left(\frac{\mathrm{d} S}{\mathrm{~d} u}\right), \quad M(x)=\int_{-\mu}^{1-\mu} \exp \{x \xi\} \varphi(\xi) \mathrm{d} \xi .
$$

\subsection{General case: random exchange rate and non-linear equation for the pdf}

To account for the entire spectrum of time scales we now assume that the exchange parameter $\gamma_{n}$ is a stochastic variable. It is convenient to write

$$
\gamma_{n}=\frac{\lambda}{1+n}\left(1+\varepsilon_{n}\right)
$$

where $\varepsilon_{n}$ is the sequence of zero mean, independent random variables. Since $0 \leq \gamma_{n} \leq 1$, it follows from Eq. (2.22) that

$$
-1 \leq \varepsilon_{n} \leq \frac{1+n}{\lambda}-1 .
$$

The decay of the variance of the exchange rate, $\sigma_{\gamma}^{2}(n)$, can be determined as

$$
\sigma_{\gamma}^{2}(n)=\left\langle\left(\gamma_{n}-\left\langle\gamma_{n}\right\rangle\right)^{2}\right\rangle=\frac{\lambda^{2}\left\langle\varepsilon_{n}^{2}\right\rangle}{(1+n)^{2}}
$$


When both $\varepsilon_{n}$ and $\xi_{n}$ are random variables, we have to specify the joint probability density for $\varepsilon$ and $\xi$. If we denote it by $\Psi(n, \varepsilon, \xi)$, then the forward Kolmogorov equation for $p(n, u)$ takes the form

$$
p(n+1, u)=\int_{-\mu}^{1-\mu} \int_{-1}^{\frac{1+n}{\lambda}}-1
$$

This equation follows from the stochastic difference equation for the Markov jump process

$$
u_{n+1}=u_{n}-\frac{\lambda}{1+n}\left(1+\varepsilon_{n}\right)\left(u_{n}-\xi_{n+1}\right) \quad \text { for } \quad-\mu \leq u_{n} \leq 1-\mu
$$

and its inverse function

$$
u_{n}=\frac{(n+1) u_{n+1}-\lambda(1+\varepsilon) \xi_{n+1}}{1+n-\lambda(1+\varepsilon)} .
$$

Since $\gamma_{n}$ is determined by the random scalar dissipation rate, it is natural to assume that $\gamma_{n}$ and $\xi_{n}$ are independent. Moreover, the density for $\xi_{n}$ must be related to $p(n, u)$. The simplest choice would be to assume $p(n, \xi)$ which gives $\Psi(n, \varepsilon, \xi)=p(n, \xi) \psi(n, \varepsilon)$ and the generalized non-linear integral equation for $p(n, u)$ can be written as

$$
p(n+1, u)=\int_{-\mu}^{1-\mu} \int_{-1}^{\frac{1+n}{\lambda}-1} \frac{n+1}{1+n-\lambda(1+\varepsilon)} p\left(n, \frac{(n+1) u-\lambda(1+\varepsilon) \xi}{1+n-\lambda(1+\varepsilon)}\right) p(n, \xi) \psi(n, \varepsilon) \mathrm{d} \varepsilon \mathrm{d} \xi
$$

for $n=1,2,3, \ldots$. It follows from Eq. (2.24) that the variance of the exchange rate $\sigma_{\gamma}^{2}(n)$ decays as $n^{-2}$. In the subsequent sections we assume for simplicity that the variance $\sigma_{\varepsilon}^{2}=\left\langle\varepsilon_{n}^{2}\right\rangle$ is constant.

We expect that the variance of a passive scalar $\sigma_{c}^{2}(n)=\left\langle\left(c_{n}-\mu\right)^{2}\right\rangle=\left\langle u_{n}^{2}\right\rangle$ behaves as $n^{-\alpha}$ for large $n$. Taking the analytical solution of the simplified model (2.5) into account, we anticipate a strong dependence of $\alpha$ on the mixing intensity $\lambda$.

\section{Results}

The integral equation (2.28) describes the relaxation of an arbitrary initial density distribution of a conserved scalar $c$ to a $\delta$-distribution for large $n$. Because of the nonlinearity in $c$ and $\widetilde{c}$, the equation needs to be solved numerically for non-trivial initial conditions.

The trivial case $\lambda=0$ describes the motion of an inert particle (e.g. dye) in its surround and Eq. (2.12) has the simple solution $u_{n}=u_{1}$ for all $n$. However, if $\lambda$ is close to its upper limit, the Lagrangian particle volume experiences strong interactions with its surrounding and intuitively, it can be expected that the mixing intensity $\lambda$ has a distinct influence on the decay of $\sigma_{u}^{2}$ (see solution (2.6) to simplified equation).

The stochastic variable $\varepsilon$ influences the exchange rate $\gamma_{n}$ and the effect of its distribution on the decay exponent $\alpha$ is desirable to understand. Formally, we can express $\alpha$ as a non-explicit available function of the form $\alpha=\alpha(\lambda, \psi(n, \varepsilon))$ where $\lambda=$ $\lambda\left(\ell_{u} / \ell_{c}, \mathrm{Pe}\right.$ or Re). In the subsequent sections the behavior of Eq. (2.28) for different parameters and initial conditions is analyzed. 


\subsection{Numerical issues}

In order to advance the density $p(n+1, u)$ in time, Eq. (2.28) must be integrated over the $\xi$ - and $\varepsilon$-space which results in the overall-complexity $\mathcal{O}\left(N_{u} \times N_{\xi} \times N_{\varepsilon}\right)$ for each time step. Here, $N_{\zeta}$ denotes the number of grid points in the $\zeta$-direction. This makes the computation rather time-consuming. However, a computer code can be easily parallelized along the $u$-coordinate.

The initial density of $u$ relaxes to a $\delta$-distribution with steep gradients around $u=0$. An adaptive grid using the equidistribution principle is employed in $u$ - and $\xi$-space in order to resolve the shape of the pdf properly. In the equidistribution principle (Liseikin 1999), an initially given number of grid points is distributed in such a way that the

condition $\int_{u_{i}}^{u_{i+1}} w(u) \mathrm{d} u=\left(N_{u}-1\right)^{-1} \int_{-\mu}^{1-\mu} w(u) \mathrm{d} u$ is satisfied. The weight function $w(u)$ is a positive function measuring the variation of the solution.

\subsection{Parameters and initial conditions}

From experiments and DNS studies it is shown that the density distribution of a passive scalar can be reasonably approximated by a $\beta$-distribution. The $\beta$-distribution has nonzero probability in the interval $\left[u_{\min }, u_{\max }\right]$ and has the form

$$
\beta\left(u ;\langle u\rangle, \sigma_{u}^{2}\right)=\frac{\Gamma(a+b)}{\Gamma(a) \Gamma(b)}\left(u_{\max }-u_{\min }\right)^{1-a-b}\left(u-u_{\min }\right)^{a-1}\left(u_{\max }-u\right)^{b-1} .
$$

It is fully described by two parameters, which are functions of $\langle u\rangle$ and $\sigma_{u}^{2}$

$$
\begin{aligned}
& a=\frac{\langle u\rangle-u_{\min }}{u_{\max }-u_{\min }}\left[\frac{\left(\langle u\rangle-u_{\min }\right)\left(u_{\max }-\langle u\rangle\right)}{\sigma_{u}^{2}}-1\right], \\
& b=\frac{u_{\max }-\langle u\rangle}{u_{\max }-u_{\min }}\left[\frac{\left(\langle u\rangle-u_{\min }\right)\left(u_{\max }-\langle u\rangle\right)}{\sigma_{u}^{2}}-1\right] .
\end{aligned}
$$

The $\beta$-distribution adopts a wide range of shapes: for the maximum variance $\sigma_{u}^{2}=\mu(1-\mu)$ and zero mean a double $\delta$-distribution at $u_{\min }$ and $u_{\max }$ is approached and for small $\sigma_{u}^{2}$ a Gaussian distribution around $\langle u\rangle$ is obtained. Throughout the following simulations, this distribution is used as initial condition for $p(1, u)$.

In order to solve Eq. (2.28) we have to provide an appropriate density distribution for the random fluctuation $\varepsilon$. The time-dependent distribution of $\varepsilon$ is certainly problemdependent and unknown in the present consideration. Therefore we assume for simplicity that $\varepsilon$ has a statistically stationary distribution. A reasonable choice would be to assume that $\varepsilon$ is distributed following Eq. (3.1). A particular case is obtained if $\psi(\varepsilon)$ has a $\delta$-distribution. For the following test cases we use both distributions as prescribed probabilities. The constant parameters for all simulations of Eq. (2.28) are summarized in Table 1.

\subsection{Influence of initial distribution in $p(1, u)$}

In the first numerical experiment the dependence of the decay exponent $\alpha$ on the initial conditions in $u$ and $\varepsilon$ is investigated. Therefore, prescribed distributions with different initial variance for $\sigma_{u}^{2}(1)$ and $\sigma_{\varepsilon}^{2}$ are used (see Table 2 and Fig. 2). For this simulation $\lambda$ is chosen to be unity and $\varepsilon$ is in the range $-1 \leq \varepsilon \leq(1+n) / \lambda-1$. The maximum possible value for $\varepsilon$ is then found for $n=1$.

The decay rate of $\sigma_{u}^{2}$ and the decay exponent $\alpha$ are evaluated and plotted in Fig. 3. In the left column, the four different combinations with initial variance $\sigma_{u}^{2}(1)=0.20$ are plotted, the middle column shows the decay rate for the cases with $\sigma_{u}^{2}(1)=0.15$. In the 


\begin{tabular}{ccc}
\hline \hline Parameter & Description & Value \\
\hline$n E n d$ & number of time steps & 100,000 \\
$\mu$ & mean value & 0.5 \\
$N_{u}$ & dimension of $u$ & 101 \\
$N_{\xi}$ & dimension of $\xi$ & 101 \\
$N_{\varepsilon}$ & dimension of $\varepsilon$ & 101 \\
\hline \hline
\end{tabular}

TABLE 1. Fixed parameters used for the numerical simulation.

\begin{tabular}{ccc}
\hline \hline Run & $p(1, u)$ & $\psi(1, \varepsilon)=\psi(\varepsilon)$ \\
\hline BB1 & $\beta(0.20)$ & $\beta(0.500)$ \\
BB2 & $\beta(0.20)$ & $\beta(0.250)$ \\
BB3 & $\beta(0.20)$ & $\beta(0.125)$ \\
BB4 & $\beta(0.15)$ & $\beta(0.500)$ \\
BB5 & $\beta(0.15)$ & $\beta(0.250)$ \\
BB6 & $\beta(0.15)$ & $\beta(0.125)$ \\
\hline \hline
\end{tabular}

\begin{tabular}{ccc}
\hline \hline Run & $p(1, u)$ & $\psi(1, \varepsilon)=\psi(\varepsilon)$ \\
\hline BB7 & $\beta(0.05)$ & $\beta(0.500)$ \\
BB8 & $\beta(0.05)$ & $\beta(0.250)$ \\
BB9 & $\beta(0.05)$ & $\beta(0.125)$ \\
BD1 & $\beta(0.20)$ & $\delta(\varepsilon)$ \\
BD2 & $\beta(0.15)$ & $\delta(\varepsilon)$ \\
BD3 & $\beta(0.05)$ & $\delta(\varepsilon)$ \\
\hline \hline
\end{tabular}

TABLE 2. Combinations of initial distributions used for the parameter study: $\beta\left(\sigma_{\zeta}^{2}\right)$ $\beta$-distribution with prescribed variance $\sigma_{\zeta}^{2} ; \delta(\zeta)$ - Dirac $\delta$-function for $\zeta$.
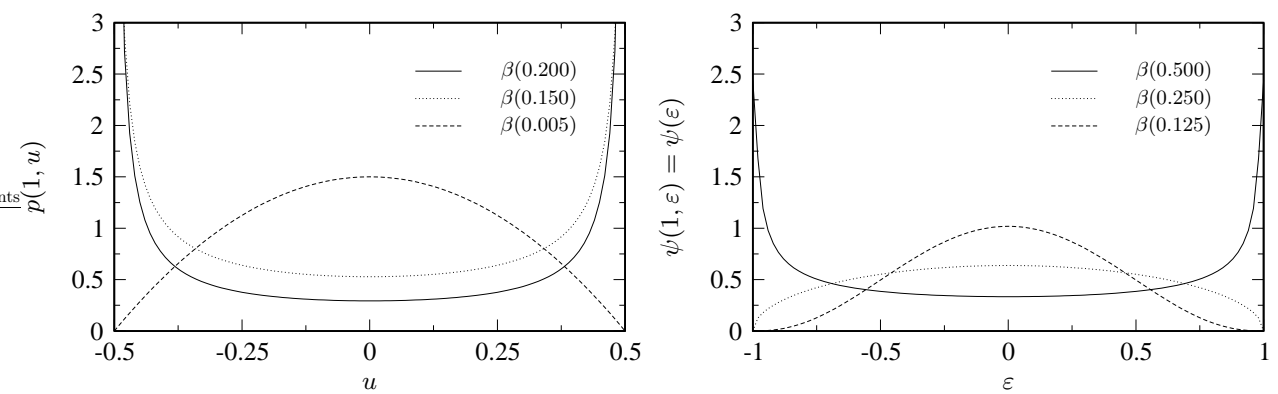

Figure 2. Initial conditions for $p(1, u)$ and $\psi(1, \varepsilon)=\psi(\varepsilon) ; \lambda=1.0$.

right column, the evolution of $p(n, u)$ is shown for the mono-modal initial $\beta$-distribution with $\sigma_{u}^{2}(1)=0.05$. From the bottom row of Fig. 3 it can be seen that $p(n, u)$ experiences strong dynamics over the initial interval, say for $n \leq 10$. Over this interval the variance does not follow the power law decay. Furthermore it can be deduced that the stochastic perturbation $\varepsilon$ reduces the decay rate of $\sigma_{u}^{2}$ if $\sigma_{\varepsilon}^{2}$ is close to $\sigma_{\varepsilon, \max }^{2}$ (see solid curves).

The influence of the initial distribution of $\varepsilon$ on the evolution of $p(n, u)$ and consequently on $\sigma_{u}^{2}(n)$ vanish for large $n$. This is most pronounced for the cases BB7-BB9 and BD3 (right column). Here the decay exponents for all of these cases collapse to a single curve, independent of $\psi(\varepsilon)$. 

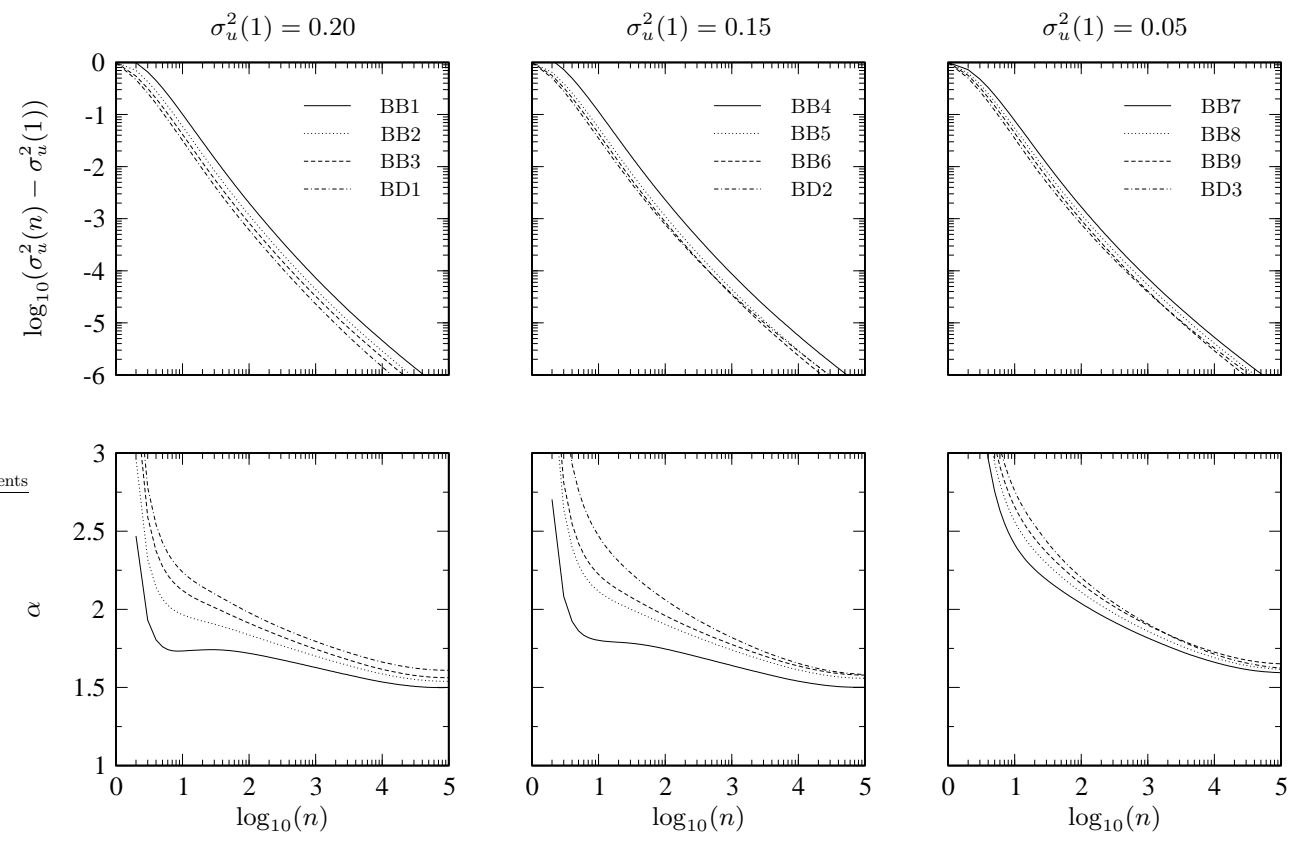

Figure 3. Evolution of $\sigma_{u}^{2}$ and decay exponent $\alpha$ for the initial $\beta$-distribution in $u$.

\begin{tabular}{lcc}
\hline \hline Run & $p(1, u)$ & $\psi(\varepsilon)$ \\
\hline Dyn1 & {$[\delta(\mu+u)+\delta(1-\mu-u)] / 2$} & $\delta(\varepsilon)$ \\
Dyn2 & $\beta(0.15)$ & $\beta(0.50)$ \\
\hline
\end{tabular}

TABLE 3. Combinations used to study dynamic behavior of $p(n, u)$ for $\lambda=0.75$.

From this simulation it can be concluded that the initially assumed distribution for $\varepsilon$ has only marginal influence on the decay exponent $\alpha$ for large $n$. During the simulation the value for $\alpha$ never reaches a steady state. The final convergence rate for $n=100,000$ is $|\mathrm{d} \alpha / \mathrm{d} n|<1.0 \times 10^{-6}$ so that $\alpha$ is in the range $1.50 \leq \alpha \leq 1.65$ for all test cases used in the present section.

\subsection{Dynamic behavior of $p(n, u)$}

In the present section we are interested in the influence of $\psi(\varepsilon)$ on the dynamics of $p(n, u)$. The transitional behavior of $\sigma_{u}^{2}$ can be retarded if the mixing intensity $\lambda$ is small. This effect was already discussed previously. In the present section the mixing intensity $\lambda=0.75$ is used.

In order to obtain insight into the transitional behavior we use a double $\delta$-distribution and a bimodal $\beta$-distribution as initial condition for $u, p(1, u)=[\delta(\mu+u)+\delta(1-\mu-u)] / 2$ and $p(1, u)=\beta(0.15)$. For $\psi(\varepsilon)=\delta(\varepsilon)$ a simple analytical solution to Eq. (2.28) can be found for the first time step $n=1$. For clarity the different combinations used here are summarized in Table 3. At $n=1$ the initial distribution for the case Dyn1 splits up into 

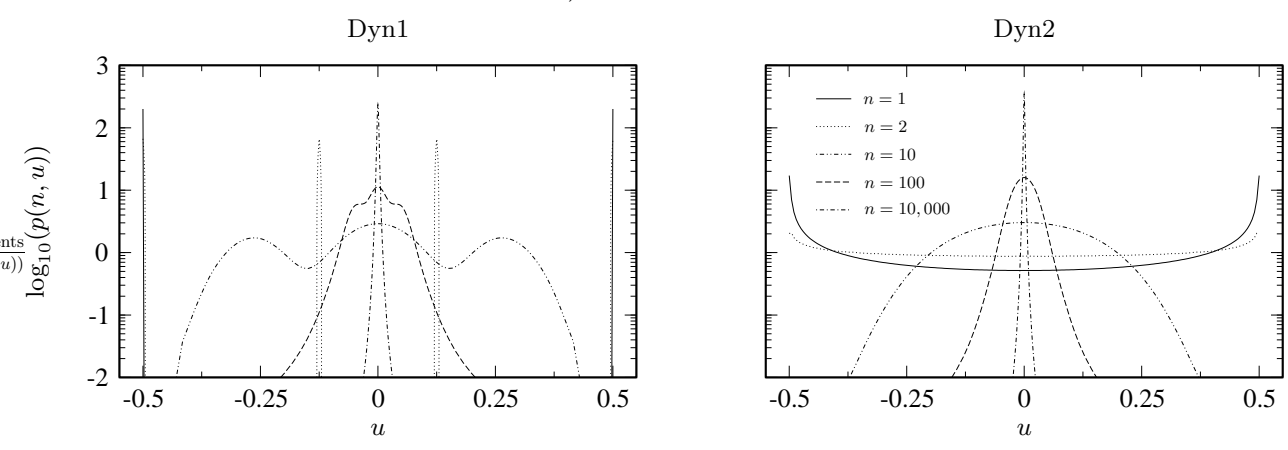

Figure 4. Transitional behavior of $p(n, u)$ for different initial conditions and mixing intensity.

\begin{tabular}{ccccc}
\hline \hline Run & $\lambda$ & $p(1, u)$ & $\psi(\varepsilon)$ & {$\left[\varepsilon_{\min }, \varepsilon_{\max }\right]$} \\
\hline LA1 & 1.50 & $\beta(0.15)$ & $\beta(0.20)$ & {$[-1,1 / 3]$} \\
LA2 & 1.00 & $\beta(0.15)$ & $\beta(0.20)$ & {$[-1,1]$} \\
LA3 & 0.75 & $\beta(0.15)$ & $\beta(0.20)$ & {$[-1,5 / 3]$} \\
LA4 & 0.50 & $\beta(0.15)$ & $\beta(0.20)$ & {$[-1,3]$} \\
LA5 & 0.25 & $\beta(0.15)$ & $\beta(0.20)$ & {$[-1,7]$} \\
\hline \hline
\end{tabular}

TABLE 4. Combinations used to study the influence of the mixing intensity $\lambda$ on the evolution of the variance.

four peaks at positions $u=\lambda(\xi-\gamma) / 2+\gamma$ with $\gamma=\{-\mu, 1-\mu\}$ and $\xi=\{-\mu, 1-\mu\}$. In the succeeding time those peaks move toward the center $u=0$ whereas the tails flatten.

An entirely different dynamic behavior is obtained for the case Dyn2. The initially smooth density function $p(1, u)$ transits rapidly to a mono-modal distribution and converges to the $\delta$-pdf. With the results of this and the previous section we can summarize that the prescribed distribution of $\varepsilon$ influences the transitional dynamics of $p(n, u)$, however its effect on the decay exponent $\alpha$ for large $n$ is insignificant.

\subsection{Mixing intensity $\lambda$}

From experiments and DNS studies it is well-known that the decay rate strongly depends on the initial ratio of the velocity and scalar length-scales, $\ell_{u}$ and $\ell_{c}$. The free parameter $\lambda$ is introduced in the exchange rate (2.22) in order to link the present model to physical processes. It needs to be emphasized that we did not attempt to reproduce any experimental or numerical data, amply and in great diversity available in the literature. Presently, we are only interested in understanding the general behavior of Eq. (2.28). It was concluded previously that the presumed form of $\psi(\varepsilon)$ has only insignificant influence on the decay rate. However, the dynamic behavior of $p(n, u)$ in the transitional range is influenced by the stochastic variable $\varepsilon$. In anticipation of the overall weak influence of $\psi(\varepsilon)$ on the decay exponent we chose arbitrarily $\psi(\varepsilon)=\beta(0.20)$ whereas $\psi(\varepsilon)$ has non-zero probability only in the interval $-1 \leq \varepsilon \leq 2 / \lambda-1$. The parameters used for the five different test cases are summarized in Table 4.

Figure 5 shows the distinct dependence of the mixing intensity on the decay of $\sigma_{u}^{2}$. 

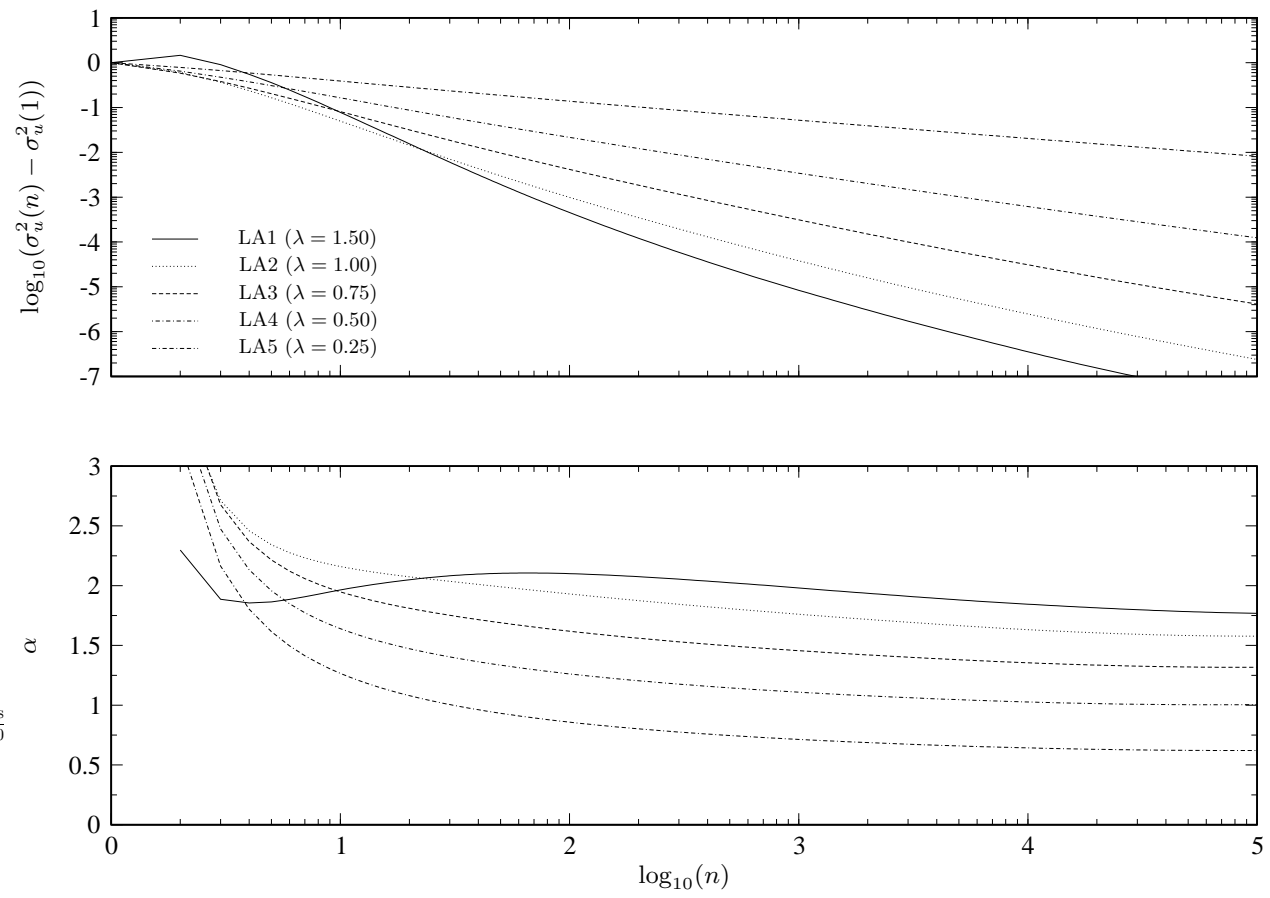

Figure 5. Decay of $\sigma_{u}^{2}$ for different mixing intensities $\lambda$.

Experiments on turbulent mixing show that the variance $\sigma_{u}^{2}$ decreases faster with increasing ratio $\ell_{u} / \ell_{c}$, until about $\ell_{u} / \ell_{c} \sim 5$. For ratios greater than that value the dependence is negligible. This tendency can be resembled by changing the mixing intensity $\lambda$. The power law exponent $\alpha$ is approximately 1.77 for high mixing intensity and decreases to about 0.62 for $\lambda=0.25$ which corresponds to an indolent mixing process. From the present findings it is difficult to specify the exact functional relation between $\lambda$ and initial conditions used in experiments. Therefore we express the mixing intensity formally as $\lambda=\lambda\left(\ell_{u} / \ell_{c}, \operatorname{Re}\right.$ or Pe $)$.

\section{Conclusion}

In the present paper we presented a robust mixing model based on the law of large numbers. The exchange rate decays as $t^{-1}$ and links the mixing model to the law of large numbers. By adding stochastic fluctuations to the exchange rate, we extend the model to the general case, taking the fluctuations of the surround into account.

The sensitivity of the model is analyzed for different initial conditions and parameters. It was shown that the initial distribution for $\varepsilon$ and $u$ has only insignificant influence on the decay rate of the variance. This is to be expected because the initial distribution of the scalar is almost never measured in experiments, assuming implicitly that either the scalar is distributed initially as a double $\delta$-function or generally disregard the dependence of the decay of $\sigma_{c}^{2}$ on the initial density distribution.

It was shown that with increasing mixing intensity the variance of the passive scalar decreases faster. The connection of our model to physical mixing processes is provided through $\lambda$, which can account for initial length-scale dependences $\ell_{u} / \ell_{c}$ and other physical effects. 


\section{REFERENCES}

Chasnov, J. R. 1994 Similarity states of passive scalar transport in isotropic turbulence. Phys. Fluids 6, 1036-1051.

CurL, R. L. 1963 Dispersed phase mixing: I Theory and effects in single reactors. AIChE journal 9, 175-181.

Dopazo, C. AND O'Brien, E. E. 1974 An approach to the autoignition of a turbulent mixture. Acta Astronautica 1, 1239-1266.

Durbin, P. A. 1982 Analysis of the decay of temperature fluctuations in isotropic turbulence. Phys. Fluids 25, 1328-1332.

FelLeR, W. 1966 An Introduction to Probability Theory and its Applications. J. Wiley \& Sons.

Knessl, C., Matkowsky, B. J., Schuss, A. \& Tier, C. 1985 An asymptotic theory of large deviations for Markov jump processes. SIAM J. Appl. Math. 46, 1006-1028.

Lesieur, M. 1997 Turbulence in Fluids. Kluwer Academic Publishers.

Liseikin, V. D. 1999 Grid Generation Methods. Springer-Verlag.

Peters, N. 2000 Turbulent Combustion. Cambridge University Press.

Pope, S. B. 2000 Turbulent Flows. Cambridge University Press.

Sabel'Nikov, V. A. \& Gorokhovski 2001 Extended LMSE and Langevin models of the scalar mixing in the turbulent flow. In Second International Symposium on Turbulence and Shear Flow Phenomena. Royal Institute of Technology (KTH), Stockholm, Sweden, June 27-29.

Villermaux, J. \& Devillon, J. C. 1972 Représentation de la coalescence et de la redispersion des domaines de ségrégation dans un fluide par un modèle d'interaction phénoménologique. in Proceedings of the Second International Symposium on Chemical Reaction Engineering, 1-13. 